\title{
Design and demonstration of tunable amplified sensitivity of AIGaN/GaN high electron mobility transistor (HEMT)-based biosensors in human serum
}

Tse-Yu Tai ${ }^{a}$, Anirban Sinha ${ }^{a}$, Indu Sarangadharan ${ }^{a}$, Anil Kumar Pulikkathodia , Shin-Li Wang ${ }^{a}$ ,Geng-Yen Lee ${ }^{\mathrm{d}}$, Jen-Inn Chyid, Shu-Chu Shieshe, Gwo-Bin Lee ${ }^{\mathrm{a}, \mathrm{b}, \mathrm{c}^{*}}$, Yu-Lin Wang ${ }^{\mathrm{a}, \mathrm{b}^{*}}$

anstitute of Nanoengineering and Microsystems, National Tsing Hua University, Hsinchu 300, Taiwan, R.O.C.

${ }^{b}$ Department of Power Mechanical Engineering, National Tsing Hua University, Hsinchu 300, Taiwan, R.O.C.

'Institute of Biomedical Engineering, National Tsing Hua University, Hsinchu 300, Taiwan, R.O.C.

${ }^{d}$ Department of Electrical engineering, National Central University, Zhongli District, Taoyuan City 320, Taiwan, R.O.C.

eDepartment of Medical Laboratory Science and Biotechnology, National Cheng Kung University, Tainan City 701, Taiwan, R.O.C

Correspondence to ylwang@mx.nthu.edu.tw

Co-correspondence to gwobin@pme.nthu.edu.tw

Supplementary information 


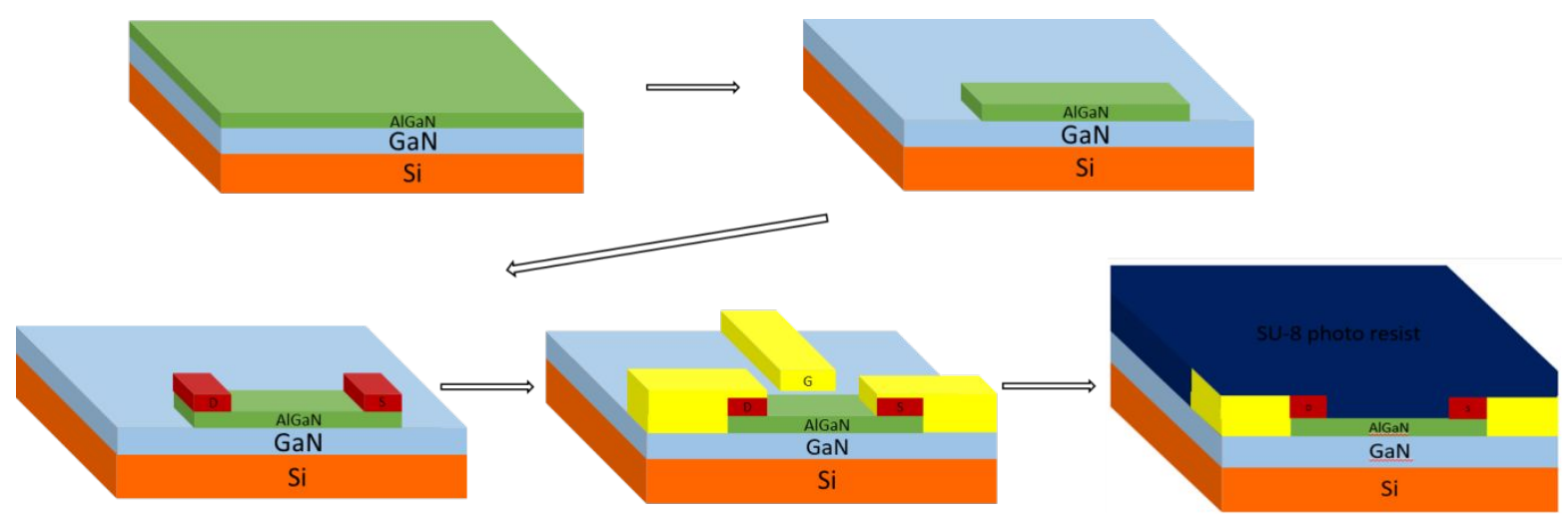

Figure S1 Fabrication of AlGaN/GaN HEMT biosensor: mesa etching of epi-wafer, ohmic contact deposition, interconnect deposition and passivation.

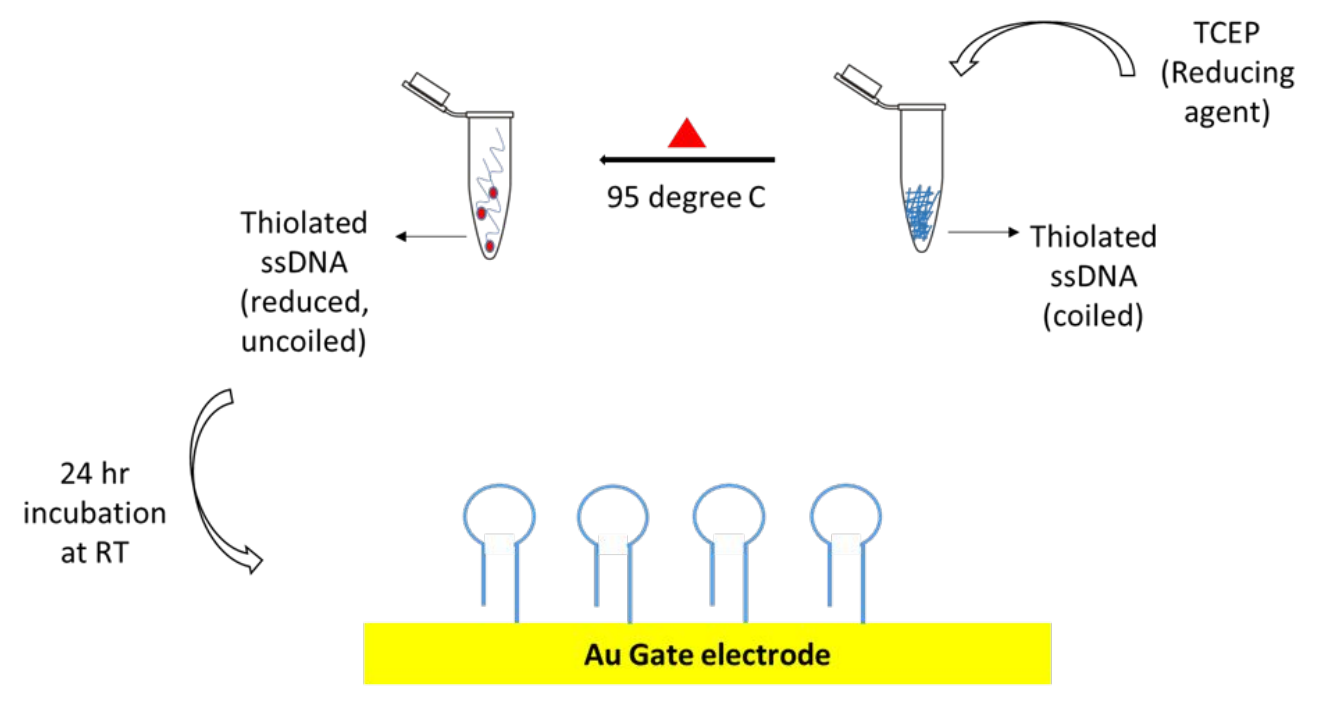

Figure S2 The schematic diagram of the aptamer immobilization process. 

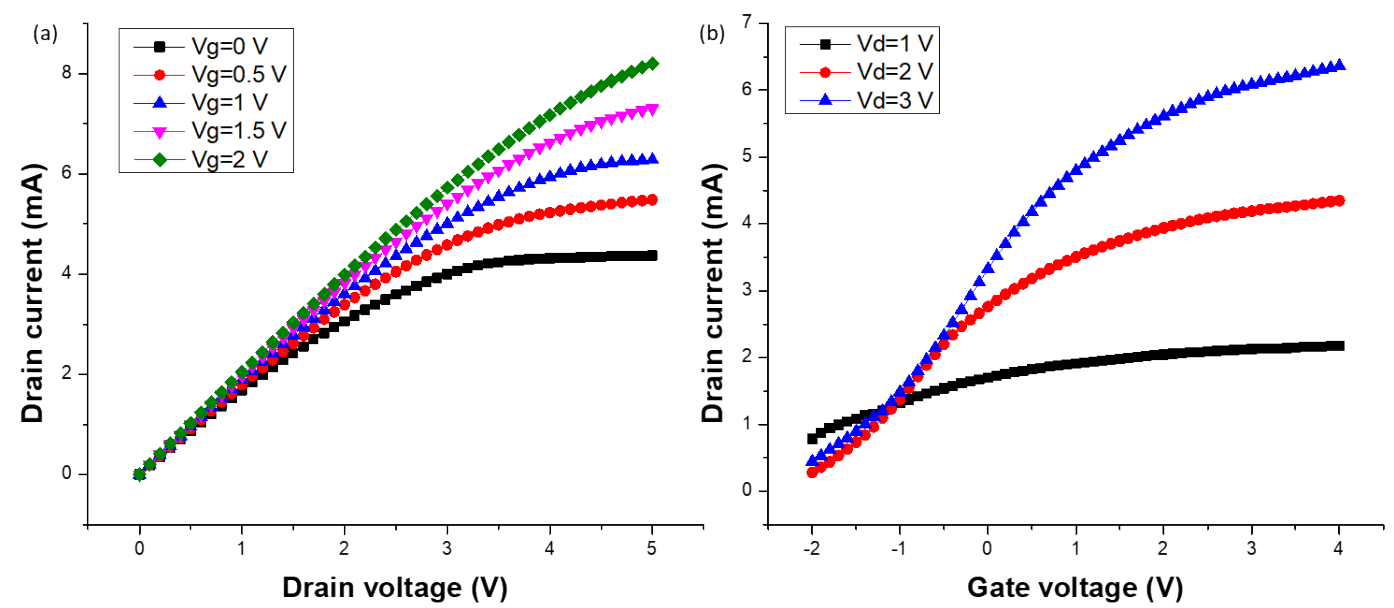

Figure S3 I-V characteristics of EDL gated AlGaN/GaN HEMT sensor (a) Drain current versus drain voltage (b) Drain current versus gate voltage.
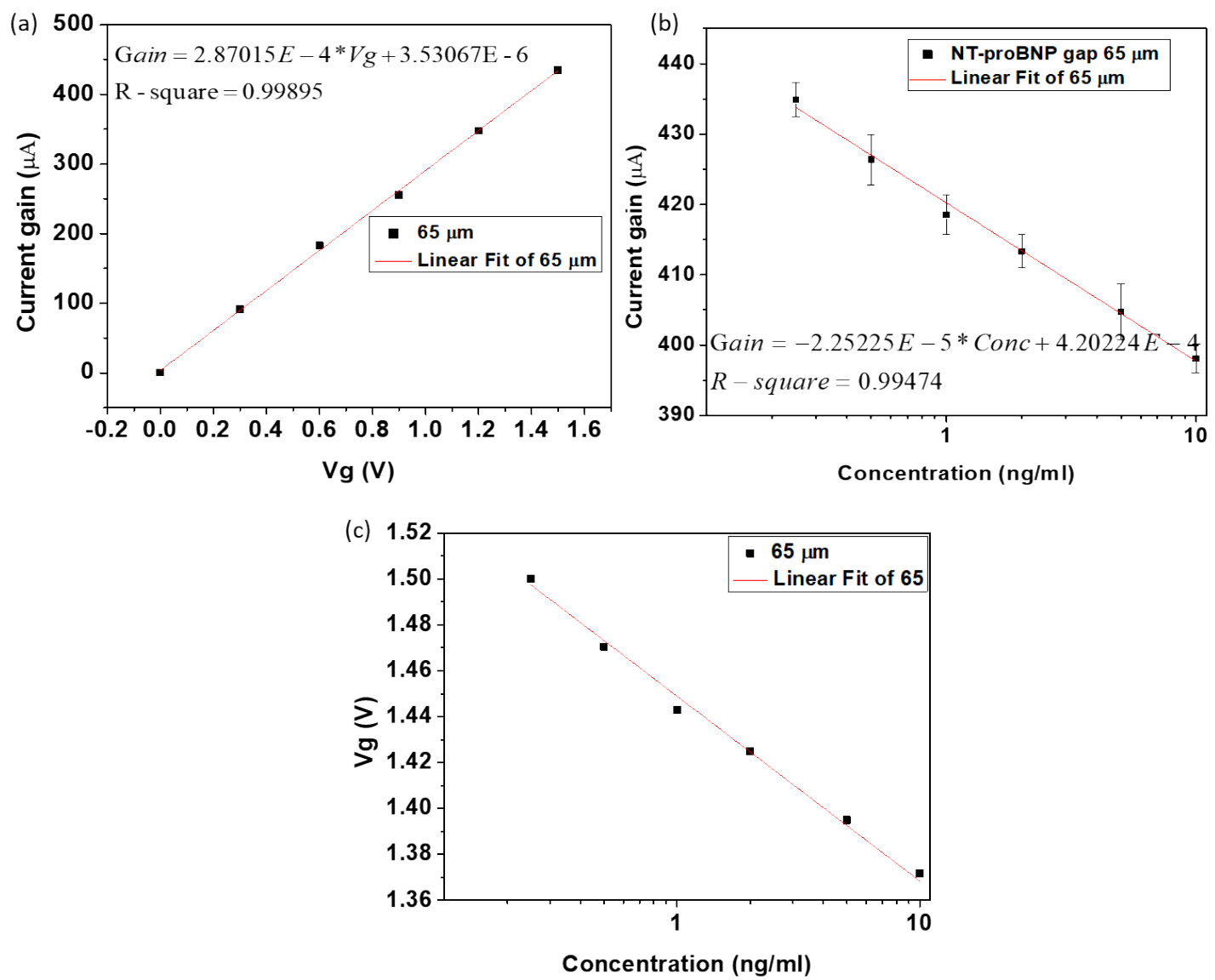
Figure S4 Sensor measurements at $65 \mu \mathrm{m}$ gap. (a) Gain versus Vg graph obtained at zero NTproBNP concentration (b) Gain versus NT-proBNP concentration obtained at $1.5 \mathrm{~V} \mathrm{Vg}$ (c) The effective Vg versus log NT-proBNP concentration graph.

(a) After testing in $1 \mathrm{XPBS}$ with $4 \% \mathrm{BSA}$

(b) After testing in clinical serum
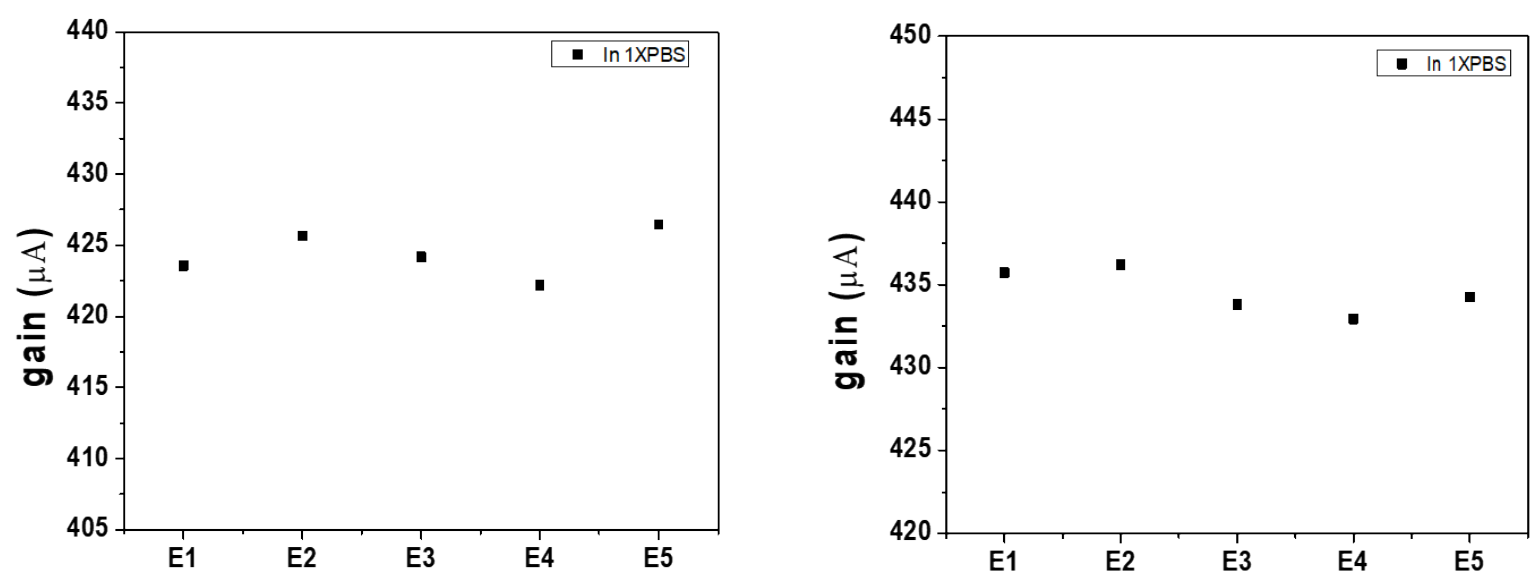

Figure S5 (a) Sensor baseline after testing in 1X PBS with 4\% BSA. E1 through E5 in $x$ axis represent the current gain values in 1 X PBS after testing $0.25,0.5,1,2,5$ and $10 \mathrm{ng} / \mathrm{mL}$ of NTproBNP in 1X PBS with 4\% BSA (b) Sensor baseline after testing in untreated human serum samples. E1 through E5 in $\mathrm{x}$ axis represent the current gain values in 1X PBS after testing 0.2239, $0.5076,1.1050,2.7470,4.6210$ and $11.4970 \mathrm{ng} / \mathrm{mL}$ of NT-proBNP in serum. 